\title{
Prospección y caracterización del yacimiento de cobre, en la vereda Los Cueros, Villanueva, departamento de La Guajira-Colombia
}

\author{
Prospection and characterization of copper deposit in Los Cueros village, \\ Villanueva, La Guajira's department-Colombia
}

\author{
Dino Carmelo Manco Jaraba ${ }^{1 *} \quad$ Kevin Rafael Ariño Díaz ${ }^{2} \quad$ Elías Ernesto Rojas Martínez ${ }^{3}$ \\ Recibido 05 de septiembre de 2017, aceptado 02 de junio de 2018 \\ Received: September 05, 2017 Accepted: June 02, 2018
}

\begin{abstract}
RESUMEN
Las concentraciones de cobre exploradas en la Serranía del Perijá, se enmarcan dentro de los yacimientos estratos confinados, generalmente asociados a rocas sedimentarias y volcanogénicas, se han realizado estudios para determinar las manifestaciones, ubicarlas y asociarlas con otros afloramientos, sin tener en cuenta su composición, concentración y demás métodos para cualificar geológicamente. El objeto de esta investigación, es evaluar concentraciones de cobre, minerales asociados y morfología de las mineralizaciones. La metodología empleada para esta investigación consistió en la toma de nueve (9) muestras, entre roca huésped, zona de contacto entre roca huésped y zona mineralizada, mediante rocas fragmentadas (Rock chips); de las cuales a ocho (8) muestras se le realizaron análisis geoquímicos mediante la técnica de Absorción atómica (AAS) y la muestra restante análisis petrográficos. A partir de los análisis, se obtuvieron resultados interesantes de concentraciones de cobre, oscilando entre 1,07\% a 12,72\%. Dichos porcentajes permitieron concluir que las concentraciones son muy buenas y seguramente sí se desarrollan adecuados estudios de exploración y evaluación geológica más detallada, podrían llegar a convertirse en proyectos de gran interés económico.
\end{abstract}

Palabras clave: Alteración hidrotermal, cobre, depósito cuprífero, geología, vulcanogénico.

\begin{abstract}
The copper concentrations explored in the Serrania of Perija, are classified within strata confined deposits, usually associated with volcanogenic sedimentary rocks, studies have been conducted to determine the copper manifestation in the field of interest, locate them and associate with other existing outcrops in the Serrania of Perija, without taking into its composition, concentration and other methods of geologically qualify this copper The purpose of this investigation is to evaluate copper concentrations, associated minerals, and mineralization morphology. The methodology used for this investigation consisted in the taking of nine (9) samples, between host rock, contact zone between host rock and mineralized zone by Rock chip; of which eight (8) samples were performed geochemical analysis by atomic absorption method (ASS), and the remaining sample by analysis petrographic. From the analysis, interesting results were obtained copper concentrations, which range between 1.07\% to $12.72 \%$. These percentages allowed us to conclude that the concentrations are very good and surely if adequate exploration and geological evaluation studies are developed, they could become projects of great economic interest.
\end{abstract}

Keywords: Copper, copper deposit, geology, hydrothermal alteration, volcanogenic.

\footnotetext{
1 Dirección de Investigación. Facultad de Ingenierías. Universidad de La Guajira. Calle 24 \#17-45. Valledupar, Colombia. Email: dinomancojaraba@gmail.com

2 Fundación Universitaria del Área Andina. Carrera 24 \# 9-110. Villanueva, Colombia. E-mail: ing.kevinarino@ hotmail.com

3 Grupo de investigación GIINGEO. Facultad de Ingenierías y Ciencias Básicas. Fundación Universitaria del Área Andina, sede Valledupar. Calle 11 Nº 21-130. Valledupar, Colombia. Email: erojas@areandina.edu.co

* Autor de correspondencia
} 


\section{INTRODUCCIÓN}

En la vereda Los Cueros, ubicada en la Serranía del Perijá, municipio de Villanueva departamento de La Guajira, existen manifestaciones de cobre [1-2]; asociados a yacimientos de tipo capas rojas (sedimentos rojos volcanoclasticos de la Formación La Quinta) [3]. Mediante recolección y análisis acompañada con una exploración superficial de las zonas mineralizadas, realizada por el semillero de investigación geológico minero de la Fundación Universitaria del Área Andina sede Valledupar, se logró tomar nueve (9) muestras, para la realización de un (1) análisis petrográfico y ocho (8) análisis de absorción atómica (ASS), con el fin de establecer paragénesis mineral, ambiente de formación, descripción de la roca huésped y concentraciones de cobre respectivamente, para así obtener mayor información que conlleve a investigaciones más profunda que permitan establecer, concentraciones, procesos genéticos de formación de las rocas huésped y mineralizaciones.

\section{LOCALIZACIÓN}

El estudio se efectuó en la vereda de Los Cueros, municipio de Villanueva, departamento de La Guajira, en una zona comprendida dentro de las siguientes coordenadas geográfica, de datum MAGNA-SIRGAS, comprendida entre norte: 1.665 .000 a $1.670 .000 \mathrm{y}$ este 1.128.000 a 1.132.000. (Figura 1).

\section{MATERIALES Y MÉTODOS}

Se identificaron varios afloramientos de cobre en la vereda Los Cueros, recolectándose 9 muestras en diferentes puntos, por el método de Roca fragmentadas (Rock Chips), que consiste en arrancar fragmentos o esquirlas de roca mineralizada, golpeando con un martillo manual o neumático en lugares regularmente distribuidos o donde se presenta la mineralización, de acuerdo con una malla, de tal manera que las porciones de rocas sean obtenidas sin contaminación (Tabla 1).

Ocho (8) muestras fueron analizadas por la técnica de absorción atómica (ASS), en el laboratorio de química y consultas industriales de la Universidad Industrial de Santander-UIS, sede de Bucaramanga para la identificación de cobre. La espectroscopia de absorción atómica es el método más empleado

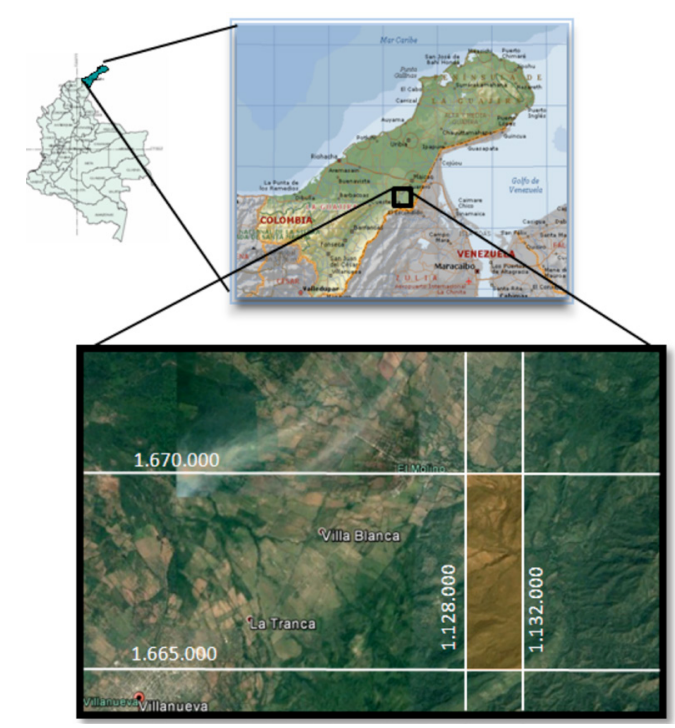

Figura 1. Localización del área estudiada.

para la determinación de metales, la muestra es pulverizada luego convertida en muestra solución que es directamente aspirada a una llama de flujo laminar. La llama tiene como función generar átomos en su estado fundamental, de los elementos presentes en la solución muestra, temperaturas cercanas a $\operatorname{los} 1,500-3,000^{\circ} \mathrm{C}$ son suficientes para producir la atomización de un gran número de elementos, los que absorberán parte de la radiación proveniente de la fuente luminosa, el número de átomos generados en su estado fundamental en la etapa de atomización determinará la cantidad de radiación absorbida; la concentración de un elemento en la muestra, se determina por comparación de la absorbancia de la solución muestra con la absorbancia de soluciones estándar de concentración conocida. Para la descripción petrográfico (1) una muestra fue seleccionada para elaboración de sección delgada, el análisis fue elaborado a partir del conteo de 300 puntos por placa, siguiendo la metodología de Le Maitre (2002) [4] (Figura 2), en el laboratorio de la Universidad Nacional- UN, sede Medellín.

\section{MARCO GEOLÓGICO REGIONAL GEOLOGÍA REGIONAL}

En el contexto regional el área de estudio hace parte del complejo rocoso conocido como terreno Perijá, [5-6]; localizado en el extremo nororiental del territorio 
Tabla 1. Muestreo y análisis efectuado.

\begin{tabular}{|c|c|c|l|}
\hline Muestra & Coordenada Este & Coordenada Norte & Análisis realizado \\
\hline M1 & 1128887 & 1661439 & Absorción Atómica \\
\hline M2 & 1128899 & 1661442 & Absorción Atómica \\
\hline M3 & 1128938 & 1661448 & Absorción Atómica \\
\hline M4 & 1128904 & 1661455 & Absorción Atómica \\
\hline M5 & 1128907 & 1661468 & Absorción Atómica \\
\hline M6 & 1128899 & 1661476 & Absorción Atómica \\
\hline M7 & 1128884 & 1661475 & Absorción Atómica \\
\hline M8 & 1128884 & 1661455 & Absorción Atómica \\
\hline M9 & 1128912 & 1661461 & Petrográfico \\
\hline
\end{tabular}

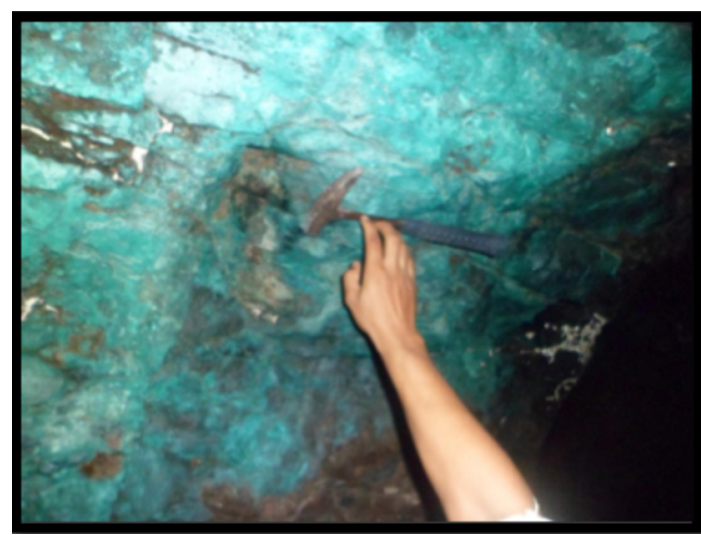

Figura 2. Reconocimiento de las zonas mineralizadas.

Colombiano, caracterizado fundamentalmente por rocas metasomáticas, filitas, esquistos pre Devónicos; intrusivos hipoabisales calco alcalinos y una potente secuencia sedimentaria clástica y bioclástica Devónica, Carboniana y Pérmica; volcanoclásticas, Juratriásicas, secuencias marinas Cretácicas y sedimentos continentales Terciarios [7-10].

\section{LITOESTRATIGRAFÍA}

Las unidades litológicas aflorantes en el área de estudio son las siguientes:

\section{ROCAS METAMÓRFICAS (P3M)}

Conjuntos de rocas pertenecientes a la facies de esquistos verdes, filitas principalmente, esquistos, cuarcitas y meta arcosas reconocidas por Forero 1972; quien las denomino como Metasedimentitas de Manaure, ubicándolas como paleozoico inferior.

\section{SEDIMENTITAS PALEOZOICAS (P3CD)}

Se pueden agrupar en esta secuencia rocas pertenecientes al Devoniano, Carboniano y Pérmico teniendo en cuenta la dificultad de separarlos por sus características litológicas sin la ayuda de consideraciones paleontológicas [11]; establece en el Perijá las siguientes secuencias de acuerdo a los periodos antes mencionados.

\section{Devoniano}

Constituido por cuatros conjuntos identificables; conglomerados basal, arenisca ferruginosa, areniscas micáceas de color verde y lutitas arenosas calcáreas arenosas [11].

\section{Carboniano}

Litológicamente está conformado por conglomerados y arcillolitas arenosas [12].

\section{Pérmico}

En el Pérmico se manifiestan areniscas, lutitas o shales y areniscas calcáreas, en la mitad superior por margas y calizas [13].

\section{FORMACIÓN LA QUINTA (Jq)}

El conjunto litológico de mayor extensión en el área investigada, para su descripción general se remite a la presentada por Forero, 1972 [11]; en la forma siguiente:

Conjunto A: compuesto por conglomerados y areniscas rojas estratificadas en capas de espesores variables, los conglomerados con espesores de 30 metros están constituidos por cantos de areniscas y en algunos casos por cantos calcáreos dentro de una matriz arenosa rojiza, 
hacia arriba predominan las areniscas con bandas conglomeráticas delgadas [11].

Conjunto B: conformado por areniscas de grano fino, de color rojo purpura y frecuentemente con estratificación cruzada e intercalaciones de arcillolitas. En este conjunto denota la carencia absoluta de conglomerados y rocas volcánicas, se le calcula un espesor de 100 metros [11].

Conjunto C: establece su límite allí donde afloran nuevamente rocas volcánicas, las Tobas forman delgadas intercalaciones de areniscas, hacia arriba se reconocen conglomerados de forma lenticular en secuencia grano decreciente lentamente a arenisca [11].

Conjunto D: se componen exclusivamente de vulcanitas acidas, riolitas o sus tobas correspondientes, parecen ser la roca que Radelli 1962 [14] señaló como riodacita. Con la presencia de estas rocas volcánicas en este conjunto termina el vulcanismo Jurásico en las rocas suprayacentes Cretácicas fundamentalmente no se ha comprobado ningún tipo de actividad volcánica. [7-8] (Figura 3).

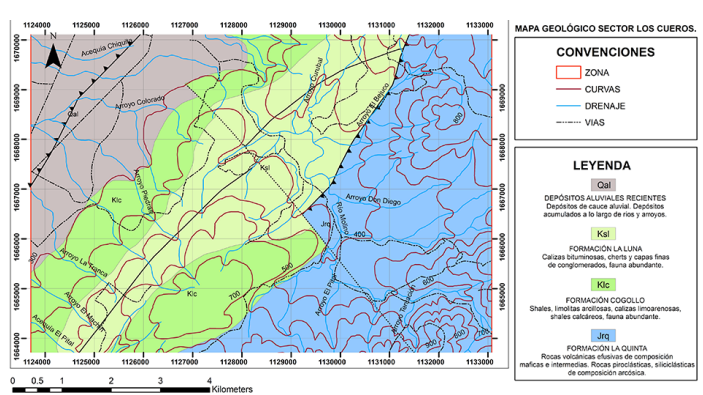

Figura 3. Mapa geológico sector Los Cueros. Tomado de Ingeominas, 2003.

\section{RESULTADOS Y DISCUSIÓN}

A partir del análisis petrográfico efectuado a la muestra (M-9), se obtuvieron las siguientes descripciones:

\section{MACROSCÓPICAMENTE}

La muestra estudiada (M-9), presentó resultados que predicen que es una muestra compacta, de color gris claro y con moteados verdes en su interior, con algunas bandas claras, eferveciendo al contacto con $\mathrm{HCl}$. Los puntos verdes corresponden a malaquita, crisocola, clorita, y epidota, se observa evidencia de transporte de fluido por los poros de la roca, así como alteración por silificación. (Figura 4).

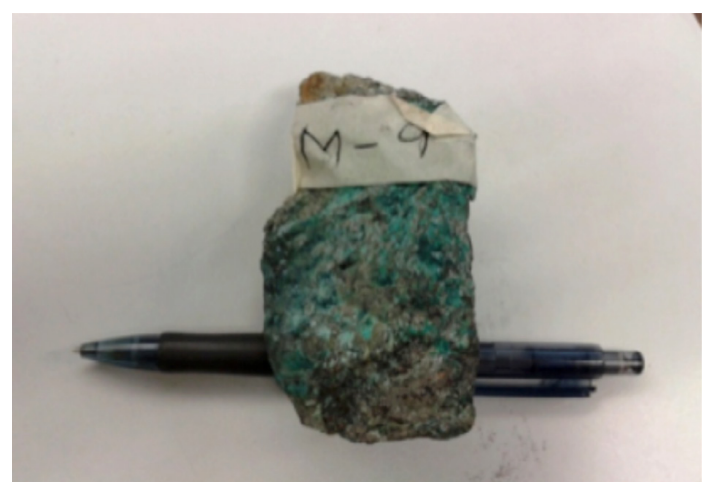

Figura 4. Muestra M-9.

\section{MICROSCÓPICAMENTE}

Se pueden observar tres dominios en la muestra, uno detrítico con mayor proporción de clastos que matriz, otro con mayor proporción de matriz que clastos y otro carbonatado.

El dominio detrítico presenta una relación clastomatriz-cemento 55\%-40\%-5\%, constituido por clastos de composición aproximada de: $40 \%$ de cuarzo $(\mathrm{Qz}), 20 \%$ de feldespato $(\mathrm{Fd}), 15 \%$ clorita (Cl), $8 \%$ de minerales opacos (Mo), $5 \%$ de micas (Mi) entre los cuales está la moscovita y cristales oxidados de biotita, $10 \%$ de un mineral azuloso que posiblemente corresponde a malaquita y epidota $2 \%$. Por lo tanto, la muestra se clasifica según Folk, 1974 [15], y Pettijohn, 1973 [16]; como Grauvaca Feldespática (Figura 5).

El dominio con mayor proporción de matriz que clastos, tiene una relación clastos- matriz-cemento diferente en porcentaje y aproximadamente de 30\%-65\%-5\%. Las composiciones de los clastos corresponden a: plagioclasas (pl) $50 \%$, clorita $(\mathrm{Cl})$ : $20 \%$, cuarzo $(\mathrm{Qz}) 10 \%$, malaquita $10 \%$, fragmentos líticos (lt) $9 \%$ y epidota $1 \%$. Tanto la matriz como el cemento de éste dominio son los mismos del dominio anterior (Figura 6). 


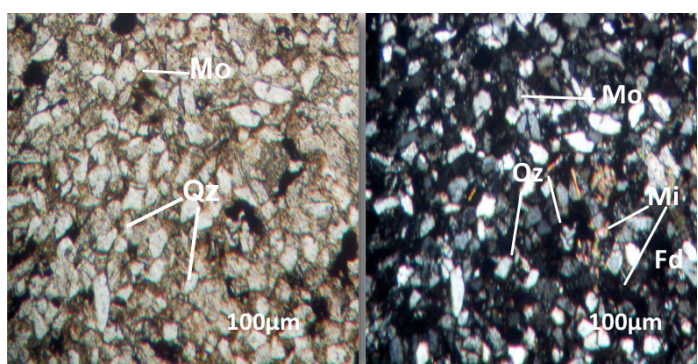

Figura 5. Microfotografía en luz transmitida. Dominio clastos-matriz.

El dominio de carbonatos es el de menor proporción en la muestra y se encuentra en contacto abrupto con el material detrítico. Presenta cristales de carbonato (Co) de tamaño aproximado de $1.2 \mathrm{~mm}$, algunos carbonatos muestran alto grado de disolución, en los cuales, se presentan cristales de mica alterada (moscovita) (Figura 7).

Los estudios geoquímicos por análisis de absorción atómica para determinación de cobre, arrojaron resultados prometedores en la medida que se logró establecer que la concentración de cobre existente en el afloramiento localizado en la vereda Los Cueros (Serranía del Perijá), poseen porcentajes que oscilan entre $1,07 \%$ a $12,72 \%$, siendo las muestras M-5, M-8, M-3 y M-7 las de mayor concentración de cobre, con porcentajes de $6.36 \%, 9,26 \%, 9,78 \%$ y $12,72 \%$ respectivamente (Figura 8). Porcentajes que promediados dan un resultado de $7 \%$ de concentración de cobre; así como la tendencia de incrementos de concentraciones hacia el SW de la Serranía de Perijá dentro de la Formación La Quinta (Figura 9), guardando concordancia con las minealizaciones en el sector de San Diego, Cesar [8]; teniendo en cuenta los conocimientos aportados por la geoquímica y la geología, [17-18]; según Fauer 1998 [19], las concentraciones de $\mathrm{Cu}$ en rocas areniscas como es el caso de estas mineralizaciones están en el orden de $3.9 \%$, considerándose anómala cuando el factor de enriquecimiento esta cuatro veces por encima de su concentración normal en la corteza, que es del 200 ppm, las concentraciones obtenidas en las mineralizaciones aflorantes en el sector de Los Cueros oscilan entre 10.700 y 120.000 ppm, muy por encima del factor de enriquecimiento, por lo tanto son prometedoras para ejecutar estudios de exploración y evaluación a mayor profundidad que permitan establecer su génesis y tipo de yacimientos,

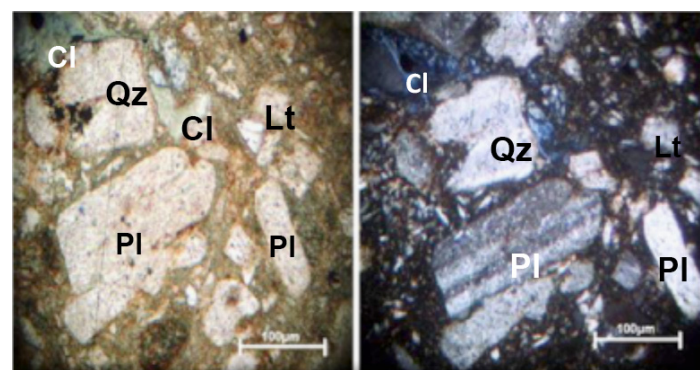

Figura 6. Microfotografía en luz transmitida. Dominio clastos-matriz.
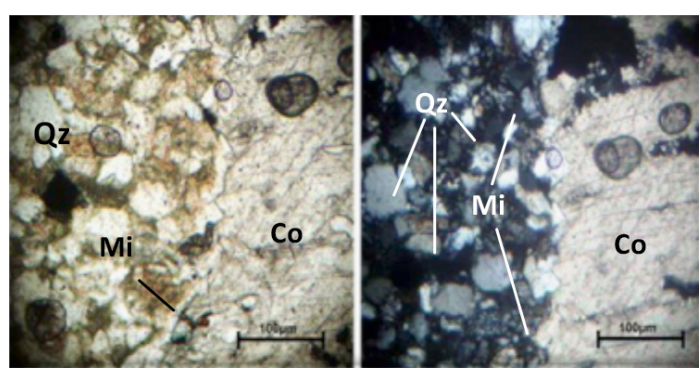

Figura 7. Microfotografía en luz transmitida. Dominio carbonatado - dominio clastos - matriz.

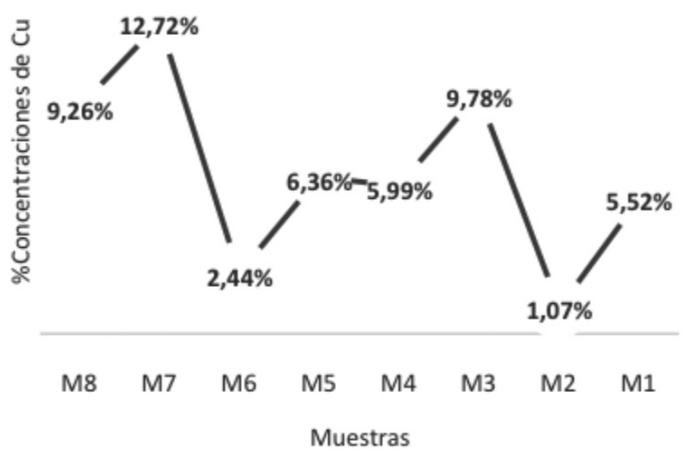

Figura 8. Muestras con sus respectivos porcentajes de cobre.

pudiéndose convertir en un proyecto de interés económico.

\section{CONCLUSIONES}

Los resultados de espectrometría de absorción atómica para cobre permiten establecer una tendencia de anomalía geoquímica hacia la parte SW de la vereda los Cueros, con incremento de concentraciones y zonas más mineralizada guardando correlación 


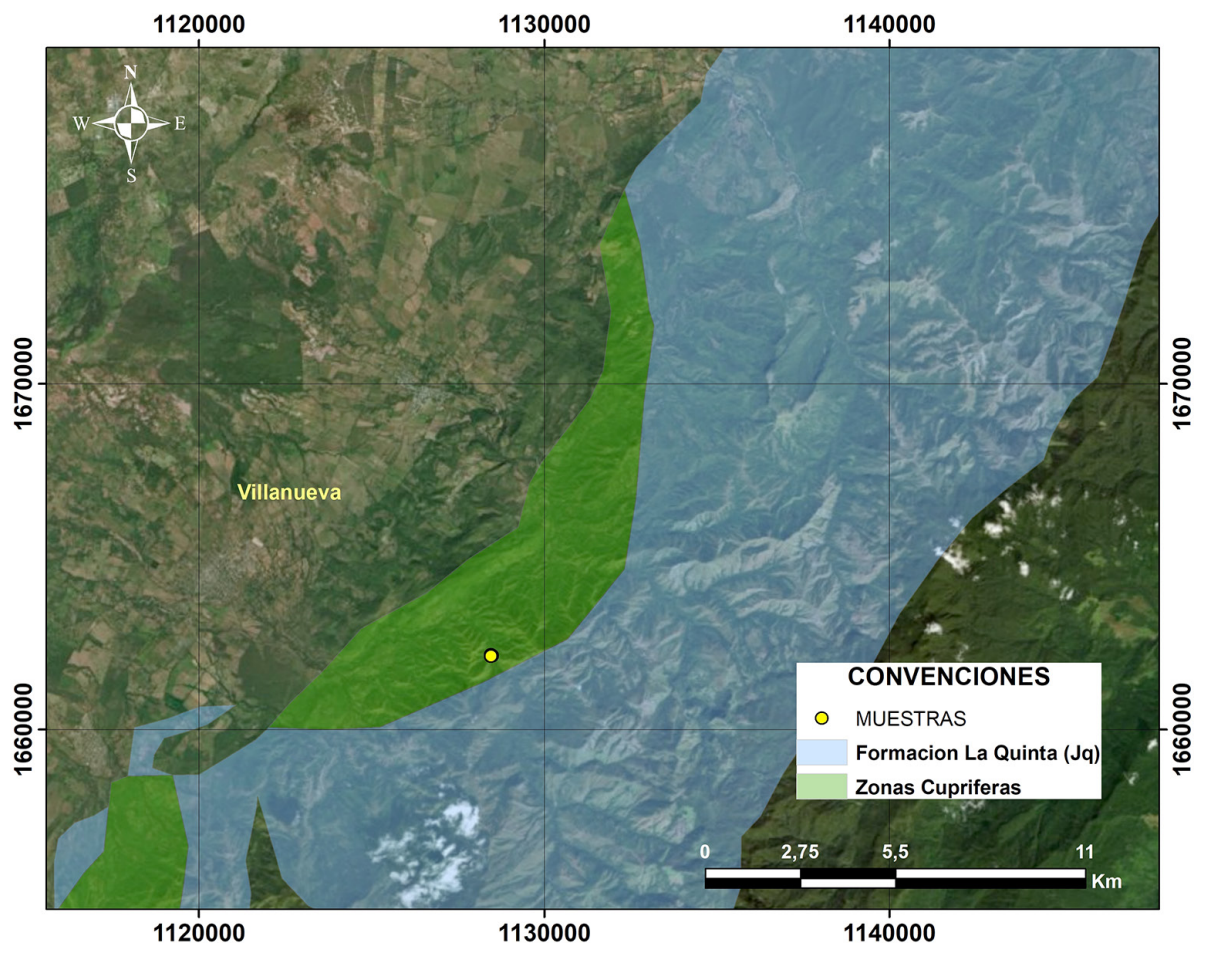

Figura 9. Mapa de la Formación La Quinta y zonas cupríferas, sector Los Cueros, Villanueva, La Guajira.

genética con otras manifestaciones a lo largo de la Serranía del Perijá dentro de la Formación La Quinta.

De acuerdo a las observaciones y la relación existente entre la mineralización con la roca caja, se puede deducir que este tipo de yacimiento correspondería a un estrato confinado embebido en una secuencia sedimentaria constituida por areniscas feldespáticas en las cuales se observan evidencias de impregnaciones por fluidos que se transportaron a través de la porosidad de la roca, creando la mineralización y generando una alteración hidrotermal a través del proceso de silificación. Los minerales que se presentan en paragénesis en la mineralización son supergenos, malaquita, azurita, crisocola, clorita, plagioclasas, carbonatos y cuprita diseminada en masas cuarzo epidóticas.

La mineralización presenta una concentración que oscilan entre $1,07 \%$ a $12,72 \%$, siendo las muestras M-5, M-8, M-3 y M-7 de las 8 muestras recolectadas las de mayor concentración de cobre, con porcentajes de $6,36 \%, 9,26 \%, 9,78 \%$ y $12,72 \%$ respectivamente, promediando una concentración de 7\% de cobre. Resultado que es apreciable, siendo esta investigación la primera en establecer valores cuantitativo a estas mineralizaciones ya que estudios anteriores solo indicaban presencia de mineralizaciones, no obstante la densidad de la muestra y la pauta de la misma no da para determinar una concentración general del yacimiento por lo cual se incentiva a que se realicen estudios de exploración y evaluación más extensos, para así llegar a conocer génesis de las mineralizaciones y tipo de yacimiento.

El análisis petrográfico determinó la existencia de tres dominios, uno detrítico con mayor proporción de clastos que matriz, otro detrítico con mayor proporción de matriz que clastos y uno carbonatado; los cuales evidencia en primera instancia los procesos sedimentarios que originaron la roca caja, y luego la actividad magmática que permitió el enriquecimiento en menas de cobres mediante fluidos hidrotermales. por lo consiguiente se puede inferir sin ser concluyente que las mineralizaciones aflorantes en la vereda Los Cueros corresponde a un yacimiento tipo epigenético. 


\section{REFERENCIAS}

[1] G. Champentier de Ribes, P. Pagnacco, L. Radelli and G. Weecksteen. "Geología y Mineralizaciones Cupríferas en la Serranía de Perijá, entre Becerril y Villanueva", Boletin Geológico. Vol. 11 No 1-3, pp. 133-188. 1963.

[2] G. Ujueta and R. Llinas, "Reconocimiento Geológico de la parte más Septentrional de la Serranía de Perijá”, Geología Colombiana. No 17, pp. 197-209. 1990.

[3] E. Viteri. "Génesis del Cobre Nativo asociado a Rocas Volcánicas de la Formación la QuintaPerijá, Venezuela", Boletín de Geología. Vol. $13 \mathrm{~N}^{\circ}$ 24, pp. 47-82. 1978.

[4] R.W. Le Maitre (Editor), A. Streckeisen, B. Zanettin, M.J. Le Bas, B. Bonin, P. Bateman, G. Bellieni, A. Dudek, S. Efremova, J. Keller, J. Lameyre, P.A. Sabine, R. Schmid, H. Sørensen, A.R. Woolley, "Igneous rocks. A Classification and Glossary of Terms, Recommendation of the International Union of Geological Science Subcommission on the systematics of Igneous rocks", 2nd Ed. Cambridge University Press. pp. 254. 2002.

[5] Instituto de Investigación e información Geocientífica Minero Ambiental y NuclearINGEOMINAS. "Geología del departamento de La Guajira”. 1991.

[6] Instituto de Investigación e información Geocientífica Minero Ambiental y NuclearINGEOMINAS. "Memorias explicativas del mapa geológico del departamento de la Guajira”, escala 1: 250.000. pp. 1-83. 2003.

[7] C. Ortega, E. Rojas and D. Manco, "Mineralización de cobre en el sector de San Diego, Serranía del Perijá", Geología Colombiana. Vol. 37, pp. 51-62. 2012.

[8] C. Ortega, E. Rojas and D. Manco, "Depósitos estrato confinados de cobre en el municipio de San Diego, Cesar, serranía del Perijá", Revista Prospectiva. Vol. 10 No 1, pp. 28-36. 2012.
[9] F. Etayo, "Mapa de terrenos geológicos de Colombia". Pub. Geol. Esp. Ingeominas. Vol. 1, pp. 1235. 1986.

[10] E. Tourtelof and J. Vine. "Copper deposits in sedimentary and volcanogenic rocks", in Geological Survey Professional Paper 907-C, United States Government Printing Office, Washington. 1976.

[11] A. Forero, "Estratigrafía del Precretácico en el flanco occidental de la Serranía de Perijá", Geología Colombiana. Vol. 7, pp. 7-78. 1972.

[12] Corpoguajira and Biocolombia, 2011. "Estudio de factibilidad para la declaración de un área natural protegida en el Corregimiento de Cañaverales - San Juan del Cesar", 2011. [online]. Disponible en: http://www. corpoguajira.gov.co/web/attachments Joom/article/949/3.\%20RESUMEN\%20 EJECUTIVO.pdf

[13] G. García. "Estudio de secciones estructurales del área conejo, Mina el Cerrejón", Tesis de pregrado, Escuela de geología, Universidad Industrial de Santander. 2010.

[14] L. Radelli, "Acerca de la geología de la Serranía de Perijá entre Codazzi y Villanueva (Magdalena-Guajira, Colombia)", Geología Colombiana. No 1, pp. 23-41. 1962.

[15] R.L. Folk. Petrology of sedimentary rocks. Texas, Hemphill Publishing Company Austin. 1974.

[16] F.P. Pettijohn, P.E. Potter and R. Siever. Sand and sandstones. New York-Heidelberg-Berlin, Springer-Verlag. 1973.

[17] Smirnov, V.I. "Geología de Yacimientos Minerales". Ed. Mir Moscú. $1^{\text {era }}$ edición. pp. 35-39. 1982.

[18] H. Garcés. "Geología económica de los yacimientos minerales y yacimientos de Colombia”. Distribuye Ademinas, Medellín, Colombia facultad de minas M5-113. 1984.

[19] G. Faure. "Principles and applications of Geochemistry". New Jersey: Prentice Hall. Upper Saddle River, N.J. 1998. 